\title{
Equivalent charge of photons in a very dense quantum plasma
}

\author{
L. A. R I O S ${ }^{1}$ and P. K. SH UK LA A ${ }^{1,2}$ \\ ${ }^{1}$ Institut für Theoretische Physik IV, Ruhr-Universität Bochum, \\ D-44780 Bochum, Germany \\ (rios@if.uff.br) \\ ${ }^{2}$ School of Physics, University of KwaZulu-Natal, Durban 4000, South Africa
}

(Received 3 July 2007 and in revised form 20 July 2007)

\begin{abstract}
The equivalent charge of photons in dense unmagnetized and magnetized Fermi plasmas is determined through the plasma physics method. This charge is associated with the polarization of the medium caused by the ponderomotive force of the electromagnetic waves. Relations for the coupling between the electron plasma density perturbation and the radiation fields are derived for unmagnetized and magnetized plasmas, taking into account the quantum force associated with the quantum Bohm potential in dense Fermi plasmas. The effective photon charge is then determined. The effects of the ion motion are also included in the investigation.
\end{abstract}

Astronomical [1-3] and experimental [4] methods allow one to set limits on the hypothetical electric charge of photons. The existence of a small photon charge would result in charge asymmetry of the Universe and would contribute to the observed cosmic microwave background (CMB) anisotropy [5], for example. However, it is possible to define an equivalent electric charge for an intense laser pulse propagating in a plasma $[6,7]$. The laser pulse can be thought as a packet of photons, each moving with the group velocity of the laser and possessing an effective mass $m_{\mathrm{eff}}=\hbar \omega_{\mathrm{pe}} / c^{2}$, where $\hbar$ is the Planck constant divided by $2 \pi, \omega_{\mathrm{pe}}$ is the electron plasma frequency, and $c$ is the speed of light in vacuum. This is possible due to the fact that electromagnetic radiation with a large spectral width can be described as a gas of photons; in this case, phase effects are negligible and the photons moving through the plasma can be considered as point-like particles. The photon electric charge is associated with the ponderomotive force (radiation pressure) of the photons, which pushes the electrons out of the region occupied by the pulse and causes the polarization of the medium. In a homogeneous plasma, this equivalent charge induces a time-dependent electric field which moves with the photon group velocity and can eventually be measured. There is also evidence that intense magnetic fields are produced during the laser-plasma interactions [8], which can affect the propagation of photons and the transport of energy in plasmas.

During the last few decades, there has been a great deal of interest in investigating the nonlinear interactions between powerful lasers and plasmas in the hope of producing a particle accelerator at the highest energies [9]. Recent experimental 
results have reinforced this idea $[10,11]$. The plasma waves generated during the laser-plasma interaction can also be used to accelerate photons $[12,13]$; here the frequency of the radiation is continuously upshifted, in analogy with the energy gain of the trailing electrons in the original concept of plasma accelerators. The coupling between electromagnetic waves and plasmas occurs due to the effective charge that photons acquire during their propagation through the medium. The radiation-plasma coupling is also important in space and astrophysical scenarios, such as in solar and stellar atmospheres, as well as in pulsar interiors (neutron stars) and in magnetospheres. Specifically, in the interior of compact objects such as neutron stars and white dwarfs the plasma is extremely dense and highly degenerate, and the plasma's properties differ significantly from those of a classical Maxwellian plasma. In such a plasma, quantum effects caused by the tunnelling of electrons/positrons must be taken into account. In dense quantum plasmas, the equilibrium particle distribution function obeys the Fermi-Dirac statistics, and the quantum effects can be described by a quantum Bohm potential. The interest in quantum plasmas has increased during the last few years, motivated by research in micro- and nano-scale objects, such as microplasmas [14] and nanowires [15], as well as in ultrasmall semiconductor devices [16]. Haas et al. [17] presented a multistream model for an ultracold Fermi plasma which accounts for the quantum force associated with the quantum Bohm potential [18]. By using the quantum hydrodynamical model, Shukla and Stenflo [19] investigated stimulated scattering instabilities of electromagnetic waves in an ultracold Fermi plasma. Recently, Glenzer et al. [20] presented the first collective X-ray scattering measurements of plasmons in the warm dense matter regime.

As mentioned before, the nonlinear photon-plasma couplings [21] are possible due to the effective charge that photons acquire during their propagation through the plasma. Mendonça et al. [6] derived an expression for the equivalent charge of photons using a classical approach where low-intensity photons were considered. Tsintsadze et al. [7] presented a kinetic theory for the induced charge of intense photons in an unmagnetized warm plasma. Shukla et al. [22] considered the influence of an external magnetic field on the induced charge of the photons. In the present work, we determine the equivalent charge of photons propagating in a dense unmagnetized/magnetized Fermi plasma. The electron-ion Fermi plasma is described by a quantum hydrodynamical model which accounts for the quantum statistical pressure law and the quantum force involving the quantum Bohm potential. We obtain expressions for the electron number density perturbations driven by the ponderomotive force of the electromagnetic wave envelope, and derive the induced charge for the photons. We compare our results with previous expressions obtained for classical plasmas, and also discuss the influence of the ion motion on the equivalent photon charge.

The dynamics of electrostatic oscillations driven by the ponderomotive force of photons in a non-relativistic electron-ion Fermi plasma are governed by the following linearized equations

$$
\begin{gathered}
\frac{\partial n_{\mathrm{i} 1}}{\partial t}+n_{0} \nabla \cdot \mathbf{u}_{\mathrm{i}}=0, \\
m_{\mathrm{i}} \frac{\partial \mathbf{u}_{\mathrm{i}}}{\partial t}=-e \nabla \phi, \\
\frac{\partial n_{\mathrm{e} 1}}{\partial t}+n_{0} \nabla \cdot \mathbf{u}_{\mathrm{e}}=0,
\end{gathered}
$$




$$
m_{\mathrm{e}} \frac{\partial \mathbf{u}_{\mathrm{e}}}{\partial t}=e \nabla \phi-\frac{T_{\mathrm{F}}}{n_{0}} \nabla n_{\mathrm{e} 1}+\frac{\hbar^{2}}{4 n_{0} m_{\mathrm{e}}} \nabla^{2} \nabla n_{\mathrm{e} 1}+\mathbf{F}_{\mathrm{e}},
$$

and

$$
\nabla^{2} \phi=4 \pi e\left(n_{\mathrm{e} 1}-n_{\mathrm{i} 1}\right) .
$$

where $n_{\mathrm{e} 1}\left(n_{\mathrm{i} 1}\right)$ and $\mathbf{u}_{\mathrm{e}}\left(\mathbf{u}_{\mathrm{i}}\right)$ are the electron (ion) density and fluid velocity perturbations created by the ponderomotive force of the electromagnetic wave envelopes. Here, $n_{\mathrm{e} 1}\left(n_{\mathrm{i} 1}\right) \ll n_{0}$, where $n_{0}$ is the equilibrium electron number density. The second term in the right-hand side of (4) is the force due to the pressure of a zero-temperature Fermi-Dirac plasma, where $T_{\mathrm{F}}=\pi^{2} \hbar^{2} n_{0}^{2 / 3} / 4 m_{\mathrm{e}}$ is the Fermi temperature (in energy units) and $m_{\mathrm{e}}$ is the electron rest mass. The third term in (4) is the quantum force associated with the Bohm potential [17], and $\mathbf{F}_{\mathrm{e}}$ is the ponderomotive force due to the high-frequency field. The electrostatic ambipolar potential $\phi$ generated due to the nonlinear polarization effects is given by the Poisson equation (5). We consider only electrostatic modes on timescales that are either comparable to or shorter than the electron plasma period, so collisions between the electrons and ions are neglected $\left(\omega_{\mathrm{pe}} \gg \nu_{\mathrm{ei}}\right.$, where $\nu_{\mathrm{ei}}$ is the collision frequency). For an unmagnetized dense Fermi plasma, the ponderomotive force is

$$
\mathbf{F}_{\mathrm{e}}=\frac{m_{\mathrm{e}}}{2} \nabla\left|\mathbf{v}_{\mathrm{e}}\right|^{2} \equiv \frac{e^{2} \nabla\left|\mathbf{E}_{0}\right|^{2}}{2 m_{\mathrm{e}} \omega_{0}^{2}},
$$

where $\mathbf{v}_{\mathrm{e}}=e\left|\mathbf{E}_{0}\right| / m_{\mathrm{e}} \omega_{0}$ is the electron quiver velocity [21], $\mathbf{E}_{0}$ is the amplitude of the electric field associated with the electromagnetic wave packet, and $\omega_{0}$ is the frequency of the photons, which is related to the wave-vector $\mathbf{k}_{0}$ by $\omega_{0}^{2}=k_{0}^{2} c^{2}+\omega_{\text {pe }}^{2}$, where $\omega_{\text {pe }}=\left(4 \pi n_{0} e^{2} / m_{\mathrm{e}}\right)^{1 / 2}$.

We now investigate the response of the electrons to the high-frequency electromagnetic fields and consider the ions at rest. Equations (1) and (2) can then be neglected, and (5) becomes $\nabla^{2} \phi=4 \pi e n_{\mathrm{e} 1}$. From the latter and (3)-(4) we then derive

$$
\left[\frac{\partial^{2}}{\partial t^{2}}+\omega_{\mathrm{pe}}^{2}+\left(\frac{\hbar^{2} \nabla^{2}}{4 m_{\mathrm{e}}^{2}}-\frac{T_{\mathrm{F}}}{m_{\mathrm{e}}}\right) \nabla^{2}\right] n_{\mathrm{e} 1}=\frac{n_{0} e^{2}}{2 m_{\mathrm{e}}^{2} \omega_{0}^{2}} \nabla^{2}\left|\mathbf{E}_{0}\right|^{2},
$$

which shows the coupling between the electron density fluctuations and the photon field. Fourier transforming (7) and using the definition of the number density of photons as $n_{\mathrm{p}}=\left|\mathbf{E}_{0}\right|^{2} / 8 \pi \hbar \omega_{0}$, we can deduce the following expression for the radiation pressure driven electron density perturbations

$$
n_{\mathrm{e} 1}=\frac{\hbar \omega_{\mathrm{pe}}^{2} k^{2} n_{\mathrm{p}}}{m_{\mathrm{e}} \omega_{0}\left(\omega^{2}-\omega_{\mathrm{pe}}^{2}-k^{2} C_{\mathrm{se}}^{2}-k^{2} C_{\mathrm{Qe}}^{2}\right)},
$$

where $C_{\mathrm{se}}=\left(T_{\mathrm{F}} / m_{\mathrm{e}}\right)^{1 / 2} \equiv v_{\mathrm{F}}$ is the Fermi electron thermal speed and $C_{\mathrm{Qe}}=$ $\hbar k / 2 m_{\mathrm{e}}$. We can establish the relation $-e n_{\mathrm{e} 2}=q_{\mathrm{p}} n_{\mathrm{p}}$, where $q_{\mathrm{p}}$ is the equivalent photon charge. Then, we obtain from (8)

$$
q_{\mathrm{p}}=-\frac{e \hbar \omega_{\mathrm{pe}}^{2} k^{2}}{m_{\mathrm{e}} \omega_{0}\left(\omega^{2}-\omega_{\mathrm{pe}}^{2}-k^{2} C_{\mathrm{se}}^{2}-k^{2} C_{\mathrm{Qe}}^{2}\right)} .
$$

We observe that the equivalent photon charge is greater in a Fermi plasma than in a classical plasma in view of larger values of $\omega_{\text {pe }}$. If we assume

$$
\omega \gg\left(\omega_{\mathrm{pe}}^{2}+k^{2} C_{\mathrm{se}}^{2}+k^{2} C_{\mathrm{Qe}}^{2}\right)^{1 / 2},
$$


and that the phase speed $v_{\phi}$ of the ponderomotive force driven density perturbation is close to $c\left(v_{\phi}=\omega / k \approx c\right)$, expression (9) then reduces to

$$
q_{\mathrm{p}} \approx-\frac{e \hbar k_{\mathrm{pe}}^{2}}{m_{\mathrm{e}} \omega_{0}}
$$

where $k_{\mathrm{pe}}=\omega_{\mathrm{pe}} / c$. This expression agrees with the previous results obtained for classical plasmas in the same regime $[6,7,22]$. The equivalent photon charge is negative because the ponderomotive force associated with the photon distribution tends to push the plasma electrons.

We now investigate the influence of the ion motion on the effective photon charge. Actually, the ions are coupled with the electrons through the space charge field, and respond on a time-scale comparable to the ion plasma period, $\omega_{\mathrm{pi}}^{-1}=$ $\left(4 \pi n_{0} e^{2} / m_{\mathrm{i}}\right)^{-1 / 2}$. The equations governing the plasma dynamics are (1)-(5); combining (1), (2) and (5) we obtain

$$
\frac{\partial^{2} n_{\mathrm{i} 1}}{\partial t^{2}}-\omega_{\mathrm{pi}}^{2}\left(n_{\mathrm{e} 1}-n_{\mathrm{i} 1}\right)=0
$$

and neglecting the electron inertia in (4), and using (5) and the definition (6) we have

$$
\omega_{\mathrm{pe}}^{2}\left(n_{\mathrm{e} 1}-n_{\mathrm{i} 1}\right)+\left(\frac{\hbar^{2} \nabla^{2}}{4 m_{\mathrm{e}}^{2}}-\frac{T_{\mathrm{F}}}{m_{\mathrm{e}}}\right) \nabla^{2} n_{\mathrm{e} 2}=\frac{n_{0} e^{2}}{2 m_{\mathrm{e}}^{2} \omega_{0}^{2}} \nabla^{2}\left|\mathbf{E}_{0}\right|^{2} .
$$

Fourier transforming (11) and (12) and using the definition of the number density of photons, we obtain

$$
n_{\mathrm{e} 1}=\frac{\hbar \omega_{\mathrm{pe}}^{2} k^{2}\left(\omega_{\mathrm{pi}}^{2}-\omega^{2}\right) n_{\mathrm{p}}}{m_{\mathrm{e}} \omega_{0}\left[\omega_{\mathrm{pe}}^{2} \omega_{\mathrm{pi}}^{2}-\left(\omega_{\mathrm{pi}}^{2}-\omega^{2}\right)\left(\omega_{\mathrm{pe}}^{2}+k^{2} C_{\mathrm{se}}^{2}+k^{2} C_{\mathrm{Qe}}^{2}\right)\right]} .
$$

Using the relation $-e n_{\mathrm{e} 2}=q_{\mathrm{p}} n_{\mathrm{p}}$, we immediately obtain the effective photon charge in the presence of the ion motion, i.e.

$$
q_{\mathrm{p}}=-\frac{e \hbar \omega_{\mathrm{pe}}^{2} k^{2}\left(\omega_{\mathrm{pi}}^{2}-\omega^{2}\right)}{m_{\mathrm{e}} \omega_{0}\left[\omega_{\mathrm{pe}}^{2} \omega_{\mathrm{pi}}^{2}-\left(\omega_{\mathrm{pi}}^{2}-\omega^{2}\right)\left(\omega_{\mathrm{pe}}^{2}+k^{2} C_{\mathrm{se}}^{2}+k^{2} C_{\mathrm{Qe}}^{2}\right)\right]} .
$$

Considering the low-frequency limit where $\omega \ll\left(k^{2} C_{\mathrm{se}}^{2}+k^{2} C_{\mathrm{Qe}}^{2}\right)^{1 / 2}$ and $\omega_{\text {pe }}$, we obtain from (14)

$$
q_{\mathrm{p}}=\frac{e \hbar}{m_{\mathrm{e}} \omega_{0}\left(\lambda_{\mathrm{De}}^{2}+\lambda_{\mathrm{Qe}}^{2}\right)},
$$

where $\lambda_{\mathrm{De}}=C_{\mathrm{se}} / \omega_{\mathrm{pe}}$ is the Fermi plasma Debye radius and $\lambda_{\mathrm{Qe}}=C_{\mathrm{Qe}} / \omega_{\mathrm{pe}}$. The expression (15) has the same form as in $[7,22]$ for an unmagnetized classical plasma in the limit $k^{2} \lambda_{\mathrm{De}}^{2} \gg 1$, but now the quantum term $\lambda_{\mathrm{Qe}}$ due to the quantum Bohm potential in the Fermi plasma appears in (15).

Next, we investigate the effect of the external magnetic field on the equivalent charge of photons in a dense Fermi plasma. Let us consider a right-hand (lefthand) circularly polarized electromagnetic (CPEM) wave propagating parallel to an external magnetic field $\mathbf{B}_{0}=B_{0} \hat{\mathbf{z}}$. The electric field of the CPEM wave is written as

$$
\mathbf{E}_{0}=E_{0}[\hat{\mathbf{x}}+(-) i \hat{\mathbf{y}}] e^{i\left(k_{0} z-\omega_{0} t\right)}+\text { c.c., }
$$

with $\omega_{0}$ and $k_{0}$ related by $\omega_{0}^{2}=k_{0}^{2} c^{2}+\omega_{0} \omega_{\text {pe }}^{2} /\left[\omega_{0}-(+) \omega_{\text {ce }}\right]$, where $\omega_{\text {ce }}=e B_{0} / m_{\mathrm{e}} c$ is the electron gyrofrequency. The electron dynamics in the presence of the ponderomotive force in a magnetized plasma is governed by the continuity equation 
(3) and by

$$
m_{\mathrm{e}} \frac{\partial \mathbf{u}_{\mathrm{e}}}{\partial t}=e \nabla \phi-\frac{e}{c}\left(\mathbf{u}_{\mathrm{e}} \times \mathbf{B}_{0}\right)+\left(\frac{\hbar^{2} \nabla^{2}}{4 n_{0} m_{\mathrm{e}}}-\frac{T_{\mathrm{F}}}{n_{0}}\right) \nabla n_{\mathrm{e} 1}+\mathbf{F}_{\mathrm{e}},
$$

where $\mathbf{F}_{\mathrm{e}}$ is the ponderomotive force due to the high-frequency CPEM field [23]. Here we consider only field-aligned perturbations associated with the slow plasma motion. Therefore, we can write $\mathbf{F}_{\mathrm{e}}=F \hat{\mathbf{z}}$, with $[23,24]$

$$
F=\frac{1}{8 \pi n_{0}}\left\{(\epsilon-1) \frac{\partial|\mathbf{E}|^{2}}{\partial z}+\frac{k}{\omega^{2}} \frac{\partial\left[\omega^{2}(\epsilon-1)\right]}{\partial \omega} \frac{\partial|\mathbf{E}|^{2}}{\partial t}\right\}_{k=k_{0}, \omega=\omega_{0}},
$$

where $\epsilon \equiv k^{2} c^{2} / \omega^{2}=1+\omega_{\mathrm{pe}}^{2} / \omega\left[\omega_{\mathrm{ce}}-(+) \omega\right]$ is the refraction index.

First, we analyse the electron plasma response by considering stationary ions. Considering only the perturbations in the $z$-direction, we have

$$
\partial n_{\mathrm{e} 1} / \partial t+n_{0}\left(\partial u_{\mathrm{e} z} / \partial z\right)=0
$$

for the continuity equation, and (for right-hand circularly polarized photons)

$$
\begin{aligned}
\frac{\partial u_{\mathrm{e} z}}{\partial t}= & \frac{e}{m_{\mathrm{e}}} \frac{\partial \phi}{\partial z}+\left(\frac{\hbar^{2}}{4 n_{0} m_{\mathrm{e}}^{2}} \frac{\partial^{2}}{\partial z^{2}}-\frac{T_{\mathrm{F}}}{n_{0} m_{\mathrm{e}}}\right) \frac{\partial n_{\mathrm{e} 1}}{\partial z} \\
& -\frac{\omega_{\mathrm{pe}}^{2}}{8 \pi n_{0} m_{\mathrm{e}} \omega_{0}\left(\omega_{0}-\omega_{\mathrm{ce}}\right)}\left[\frac{\partial}{\partial z}-\frac{k_{0} \omega_{\mathrm{ce}}}{\omega_{0}\left(\omega_{0}-\omega_{\mathrm{ce}}\right)} \frac{\partial}{\partial t}\right]\left|\mathbf{E}_{0}\right|^{2},
\end{aligned}
$$

with $\partial^{2} \phi / \partial z^{2}=4 \pi e n_{\mathrm{e} 1}$. From the above equations, we obtain

$$
\begin{aligned}
& {\left[\frac{\partial^{2}}{\partial t^{2}}+\omega_{\mathrm{pe}}^{2}+\left(\frac{\hbar^{2}}{4 m_{\mathrm{e}}^{2}} \frac{\partial^{2}}{\partial z^{2}}-\frac{T_{\mathrm{F}}}{m_{\mathrm{e}}}\right) \frac{\partial^{2}}{\partial z^{2}}\right] n_{\mathrm{e} 1}} \\
& \quad=\frac{n_{0} e^{2}}{2 m_{\mathrm{e}}^{2} \omega_{0}\left(\omega_{0}-\omega_{\mathrm{ce}}\right)}\left[\frac{\partial}{\partial z}-\frac{k_{0} \omega_{\mathrm{ce}}}{\omega_{0}\left(\omega_{0}-\omega_{\mathrm{ce}}\right)} \frac{\partial}{\partial t}\right] \frac{\partial\left|\mathbf{E}_{0}\right|^{2}}{\partial z},
\end{aligned}
$$

which shows the coupling between the electron density fluctuations and the photon field in the presence of the external magnetic field. Fourier transforming (20) and using $n_{\mathrm{p}}=\left|\mathbf{E}_{0}\right|^{2} / 8 \pi \hbar \omega_{0}$ and the relation $-e n_{\mathrm{e} 2}=q_{\mathrm{p}} n_{\mathrm{p}}$, we derive the following expression for the equivalent photon charge in a magnetized dense Fermi plasma

$$
q_{\mathrm{p}}=-\frac{e \hbar \omega_{\mathrm{pe}}^{2} k^{2}\left(1+\alpha_{\mathrm{e}} k_{0} / k\right)}{m_{\mathrm{e}}\left(\omega_{0}-\omega_{\mathrm{ce}}\right)\left(\omega^{2}-\omega_{\mathrm{pe}}^{2}-k^{2} C_{\mathrm{se}}^{2}-k^{2} C_{\mathrm{Qe}}^{2}\right)},
$$

where $\alpha_{\mathrm{e}}=\omega \omega_{\text {ce }} / \omega_{0}\left(\omega_{0}-\omega_{\text {ce }}\right)$. In the absence of the magnetic field, (21) reduces to the expression (9), as expected. In the limit $\omega^{2} \gg \omega_{\mathrm{pe}}^{2}+k^{2} C_{\mathrm{se}}^{2}+k^{2} C_{\mathrm{Qe}}^{2}$ and with $v_{\phi}=\omega / k \approx c, k_{\mathrm{pe}}=\omega_{\mathrm{pe}} / c$ and $k / k_{0} \gg \alpha_{\mathrm{e}}$, expression (21) becomes

$$
q_{\mathrm{p}}=-\frac{e \hbar k_{\mathrm{pe}}^{2}}{m_{\mathrm{e}}\left(\omega_{0}-\omega_{\mathrm{ce}}\right)} .
$$

For the right-hand circularly polarized photons, the magnitude of the induced electric charge increases in the presence of the external magnetic field (for the lefthand circularly polarized waves, it decreases [22]). Once more, $q_{\mathrm{p}}$ attains a large value due to the extremely high value of $\omega_{\text {pe }}$ in a dense Fermi plasma.

Second, we investigate the influence of the ion motion on the effective photon charge in a magnetized dense Fermi plasma. For this purpose, we have also to consider the ion continuity and momentum equations in the $z$-direction. Neglecting 
the electron inertia in (17) and combining the equations for the electrons and ions, with $\partial^{2} \phi / \partial z^{2}=4 \pi e\left(n_{\mathrm{e} 1}-n_{\mathrm{i} 1}\right)$, we derive (11) and

$$
\begin{aligned}
& \omega_{\mathrm{pe}}^{2}\left(n_{\mathrm{e} 1}-n_{\mathrm{i} 1}\right)+\left(\frac{\hbar^{2}}{4 m_{\mathrm{e}}^{2}} \frac{\partial^{2}}{\partial z^{2}}-v_{\mathrm{F}}^{2}\right) \frac{\partial^{2} n_{\mathrm{e} 1}}{\partial z^{2}} \\
& \quad=\frac{n_{0} e^{2}}{2 m_{\mathrm{e}}^{2} \omega_{0}\left(\omega_{0}-\omega_{\mathrm{ce}}\right)}\left[\frac{\partial}{\partial z}-\frac{k_{0} \omega_{\mathrm{ce}}}{\omega_{0}\left(\omega_{0}-\omega_{\mathrm{ce}}\right)} \frac{\partial}{\partial t}\right] \frac{\partial\left|\mathbf{E}_{0}\right|^{2}}{\partial z} .
\end{aligned}
$$

Fourier transforming (11) and (23) and combining the resultant equations, we obtain

$$
q_{\mathrm{p}}=-\frac{e \hbar \omega_{\mathrm{pe}}^{2} k^{2}\left(\omega_{\mathrm{pi}}^{2}-\omega^{2}\right)\left(1+\alpha_{\mathrm{e}} k_{0} / k\right)}{m_{\mathrm{e}}\left(\omega_{0}-\omega_{\mathrm{ce}}\right)\left[\omega_{\mathrm{pi}}^{2} \omega_{\mathrm{pe}}^{2}-\left(\omega_{\mathrm{pi}}^{2}-\omega^{2}\right)\left(\omega_{\mathrm{pe}}^{2}+k^{2} C_{\mathrm{se}}^{2}+k^{2} C_{\mathrm{Qe}}^{2}\right)\right]} .
$$

In the limit where the phase speed of the excited wave is much smaller than the electron thermal speed and $k / k_{0} \gg \alpha_{\mathrm{e}}$, we can write $(24)$ as

$$
q_{\mathrm{p}}=\frac{e \hbar}{m_{\mathrm{e}}\left(\omega_{0}-\omega_{\mathrm{ce}}\right)\left(\lambda_{\mathrm{De}}^{2}+\lambda_{\mathrm{Qe}}^{2}\right)} .
$$

To summarize, we have calculated the equivalent charge of photons in an unmagnetized/magnetized dense Fermi plasma by using the plasma physics method. The electromagnetic wave packet propagating in the plasma is described as a gas of photons, and a quantum hydrodynamical model, including the effects of the quantum statistical pressure and the quantum force, is used to describe the Fermi plasma particle dynamics. The radiation pressure of the photons creates space charge electric fields, which cause the plasma polarization and induced charges. Explicit expressions for the latter are given for dense plasmas without and with the ion dynamics. Our results show that in the high-frequency limit the induced charge is significantly larger due to higher values of $\omega_{\text {pe }}$ in a dense quantum plasma. Furthermore, in the low-frequency limit the influence of the quantum Bohm potential is to reduce the magnitude of the induced photon charge. It has also been found that the presence of an external magnetic field enhances (reduces) the induced charge of right-hand (left-hand) circularly polarized photons in a dense Fermi plasma. These results can be important for the study of photon charges in degenerate astrophysical plasmas, such as those in the interior of dense neutron stars and white dwarfs, as well as for dense quantum plasmas in intense laser-solid density plasma interaction experiments and other practical systems.

\section{Acknowledgement}

This work was partially supported by CNPq (Conselho Nacional de Desenvolvimento Científico e Tecnológico), Brazil.

\section{References}

[1] Cocconi, G. 1988 Phys. Lett. B 206, 705.

[2] Raffelt, G. 1994 Phys. Rev. D 50, 7729.

[3] Kobychev, V. V. and Popov, S. B. 2005 Astron. Lett. 31, 147.

[4] Semertzidis, Y. K., Danby, G. T and Lazarus, D. M. 2003 Phys. Rev. D 67, 017701.

[5] Caprini, C. and Ferreira, P. G. 2005 J. Cosmol. Astropart. Phys. 02, 006. 
[6] Mendonça, J. T., Silva, L. O., Bingham, R., Tsintsadze, N. L., Shukla, P. K. and Dawson, J. M. 1998 Phys. Lett. A $\mathbf{2 3 9}, 373$.

[7] Tsintsadze, N. L., Mendonça, J. T. and Shukla, P. K. 1998 Phys. Lett. A 249, 110.

[8] Wilks, S. C., Kruer, W. L., Tabak, M. and Langdon, A. B. 1992 Phys. Rev. Lett. 69, 1383. Bulanov, S. V., Lontano, M., Esirkepov, T. Zh., Pegoraro, F. and Pukhov, A. M. 1996 Phys. Rev. Lett. 76, 3562.

Borghesi, M., MacKinnon, A. J., Bell, A. R., Gaillard, R. and Willi, O. 1998 Phys. Rev. Lett. 81, 112.

Clark, E. L. et al. 2000 Phys. Rev. Lett. 84, 670. Pollock, B. B. et al. 2006 Rev. Sci. Instrum. 77, 114703.

[9] Mendonça, J. T. 2001 Theory of Photon Acceleration. Bristol: Institute of Physics. Bingham, R., Mendonça, J. T. and Shukla, P. K. 2004 Plasma Phys. Control. Fusion 46, R1.

[10] Geddes, C. G. R., Tóth, Cs., van Tilborg, J., Esarey, E., Schroeder, C. B., Bruhwiler, D., Nicter, C., Cary, J. and Leemans, W. P. 2004 Nature 431, 538.

[11] Leemans, W. P., Nagler, B., Gonsalves, A. J., Tóth, Cs., Nakamura, K., Geddes, C. G. R., Esarey, E., Schroeder, C. B. and Hooker, S. M. 2006 Nature Phys. 2, 696.

[12] Wilks, S. C., Dawson, J. M., Mori, W. B., Katsouleas, T. and Jones, M. E. 1989 Phys. Rev. Lett. 62, 2600.

[13] Murphy, C. D. et al. 2006 Phys. Plasmas 13, 033108.

[14] Becker, K., Koutsospyros, A., Yin, S.-M., Christodoulatos, C., Abramzon, N., Joaquin, J. C., Brelles-Mariño, G. 2005 Plasma Phys. Control. Fusion 47, B513.

[15] Robinson, I. K., Bennett, P. A. and Himpsel, F. J. 2002 Phys. Rev. Lett. 88, 096104. Tilke, A. T., Simmel, F. C., Lorenz, H., Blick, R. H. and Kotthaus, J. P. 2003 Phys. Rev. B 68, 075311.

[16] Markowich, P. A., Ringhofer, C. A. and Schmeiser, C. 1990 Semiconductor Equations. Berlin: Springer.

[17] Haas, F., Manfredi, G. and Feix, M. 2000 Phys. Rev. E 62, 2763.

[18] Gardner, C. L. and Ringhofer, C. 1996 Phys. Rev. E 53, 157.

Shukla, P. K. and Eliasson, B. 2006 Phys. Rev. Lett. 96, 245001. Shukla, P. K. and Eliasson, B. 2007 New J. Phys. 9, 98.

[19] Shukla, P. K. and Stenflo, L. 2006 Phys. Plasmas 13, 044505.

[20] Glenzer, S. H. et al. 2007 Phys. Rev. Lett. 98, 065002.

[21] Marklund, M. and Shukla, P. K. 2006 Rev. Mod. Phys. 78, 591.

[22] Shukla, P. K., Tsintsadze, N. L., Mendonça, J. T. and Stenflo, L. 1999 Phys. Plasmas 6, 627.

[23] Shukla, P. K. and Stenflo, L. 1984 Phys. Rev. A 30, 2110.

Shukla, P. K., Rao, N. N., Yu, M. Y. and Tsintsadze, N. L. 1986 Phys. Rep. 138, 1.

[24] Karpman, V. I. and Washimi, H. 1977 J. Plasma Phys. 18, 173. 